\title{
Inappropriate prescription of allopurinol and febuxostat and risk of adverse events in the elderly: results from the REPOSI registry
}

\author{
L. Pasina • A. L. Brucato • C. D. Djade • P. Di Corato • S. Ghidoni • \\ M. Tettamanti - C. Franchi • F. Salerno • S. Corrao • A. Marengoni • \\ M. Marcucci • P. M. Mannucci • A. Nobili • REPOSI Investigators
}

Received: 15 July 2014 / Accepted: 9 September 2014 / Published online: 18 September 2014

(C) Springer-Verlag Berlin Heidelberg 2014

\begin{abstract}
Purpose To investigate the prevalence of xanthine oxidase (XO) inhibitors prescription at admission and discharge in elderly hospital in-patients, to analyze the appropriateness of their use in relation to evidence-based indications, to evaluate the predictors of inappropriate prescription at discharge and
\end{abstract}

REPOSI stands for Registry of Polytherapies SIMI (Società Italiana di Medicina Interna)

L. Pasina $(\bowtie) \cdot$ C. D. Djade $\cdot$ M. Tettamanti $\cdot$ C. Franchi $\cdot$ A. Nobili Laboratory for Quality Assessment of Geriatric Therapies and Services, Drug Information Service for the Elderly, IRCCS - Istituto di Ricerche Farmacologiche "Mario Negri", Via Giuseppe La Masa, 19, 20156 Milan, Italy

e-mail: luca.pasina@marionegri.it

A. L. Brucato $\cdot$ P. Di Corato $\cdot$ S. Ghidoni

Internal Medicine, AO Papa Giovanni XXIII, Bergamo, Italy

F. Salerno

Internal Medicine I, Policlinico IRCCS San Donato, University of Milan, Milan, Italy

S. Corrao

Dipartimento Biomedico di Medicina Interna e Specialistica, University of Palermo, Palermo, Italy

\section{A. Marengoni}

Geriatric Unit, Spedali Civili, Department of Clinical and

Experimental Sciences, University of Brescia, Brescia, Italy

M. Marcucci

Department of Internal Medicine, University of Perugia, Perugia, Italy

P. M. Mannucci

Scientific Director, IRCCS Maggiore Hospital Foundation, Milan, Italy the association with adverse events 3 months after hospital discharge.

Methods This cross-sectional study, based upon a prospective registry, was held in 95 Italian internal medicine and geriatric hospital wards. The sample included 4035 patients aged 65 years or older at admission and 3502 at discharge. The prescription of $\mathrm{XO}$ inhibitors was considered appropriate in patients with diagnosis of gout, gout nephropathy, uric acid nephrolithiasis, tophi, and chemotherapy-induced hyperuricemia. In order to evaluate the predictors of inappropriate prescription of XO inhibitors, we compared the characteristics of patients considered inappropriately treated with those appropriately not treated.

Results Among the 4035 patients eligible for the analysis, 467 $(11.6 \%)$ were treated with allopurinol or febuxostat at hospital admission and 461 (13.2\%) among 3502 patients discharged. At admission, 39 (8.6 \%) of patients receiving XO inhibitors and $43(9.4 \%)$ at discharge were appropriately treated. Among those inappropriately treated, hyperuricemia, polytherapy, chronic renal failure, diabetes, obesity, ischemic cardiomyopathy, heart failure, and cardiac dysrhythmias were associated with greater prescription of XO inhibitors. Prescription of XO inhibitors was associated with a higher risk of adverse clinical events in univariate and multivariate analysis.

Conclusions Prevalence of inappropriate prescription of $\mathrm{XO}$ inhibitors remained almost the same at admission and discharge. Inappropriate use of these drugs is principally related to treatment of asymptomatic hyperuricemia and various cardiovascular diseases.

Keywords Aged $\cdot$ Pharmacoepidemiology $\cdot$ Allopurinol 


\section{Background}

In the occurrence of gout, the culprit is the accumulation in joints of uric acid, mainly formed by the enzyme xanthine oxidase (XO) which converts hypoxanthine into uric acid, superoxide, and other oxidative-free radicals. XO inhibitors are the treatment of choice for gout, and allopurinol is used by more than 1.2 million patients in the USA and UK. Inhibition of XO by allopurinol has been suggested to be associated to various clinical benefits: improvement of endothelial dysfunction, reduction of vascular tissue oxidative stress, increase in ATP energy, and oxygenation of ischemic tissues [1]. Accordingly, allopurinol has been considered potentially useful in an array of conditions other than gout: prevention or regression of atherosclerosis [2], prevention of cardiovascular disease (CVD) in patients who had had an acute coronary syndrome (ACS) or myocardial infarction (MI), transient ischemic attacks (TIA), cerebral vascular accidents (CVA), intermittent claudication, and heart failure after MI. However, little evidence supports a role of allopurinol in the prevention of CVD and there is still an open debate regarding its role in asymptomatic hyperuricemia, because only two epidemiological and experimental studies (in heart failure and hyperuricemia) suggested a link between hyperuricemia, hypertension, and lower total mortality [3, 4], and only two small randomized controlled trials showed that allopurinol reduces the incidence of CVD $[5,6]$. On the other hand, the allopurinol hypersensitivity syndrome is rare but often occurs as a consequence of inappropriate treatment with this agent [7], so that therapeutic decisions should take into account the balance between expected benefits and potential harms. Febuxostat, a new potent non-purine-selective inhibitor of $\mathrm{XO}$, is now recommended as the first-line urate-lowering therapy for gout jointly with allopurinol according to the American College of Rheumatology guidelines [8]. Although this drug provides more selective, potent and persistent inhibition of $\mathrm{XO}$ and greater hypouricemic activity than allopurinol, there is no evidence for its benefits in CVD [9].

With this background and considering the licensed indications for XO inhibitors (management of signs and symptoms of primary or secondary gout, chemotherapy-induced hyperuricemia, and recurrent calcium oxalate stones), because little is known on the appropriateness of prescription of XO inhibitors in elderly patients hospitalized, we used REPOSI, a registry stemming from a network of Italian internal medicine and geriatric wards, in order to investigate the rate of allopurinol and febuxostat prescription at hospital admission and discharge in the elderly. Other goals were the analysis of appropriateness in relation to evidence-based drug indications, the predictors of inappropriate prescription, and its association with the risk of adverse clinical events, re-admission, and allcause mortality 3 months after hospital discharge. For the purpose of the study, the prescription of $\mathrm{XO}$ inhibitors was considered appropriate in patients with diagnosis of gout, gout nephropathy, uric acid nephrolithiasis, tophi, and chemotherapy-induced hyperuricemia.

\section{Methods}

Data collection

The Registro Politerapie SIMI (REPOSI) is a collaborative and independent, initiative of the Italian Society of Internal Medicine (SIMI), the IRCCS Istituto di Ricerche Farmacologiche Mario Negri and the IRCCS $\mathrm{Ca}$ ' Granda Maggiore Policlinico Hospital Foundation. The registry was set up in 2008 from a network of internal medicine and geriatric wards in order to collect information on hospitalized elderly patients with multimorbidities receiving multiple drugs. The first run of data collection was between January and December 2008, the second between January and December 2010, and the third between January and December 2012. To ensure an unselected population of elderly patients admitted to internal medicine wards, during four-week period, 3 months apart, the first ten patients admitted to the wards participating in the study were consecutively recruited if they were 65 years old or older. Participation was voluntary and all patients gave signed informed consent. Data collection complied fully with the Italian laws on personal data protection and Ethical Committees of each ward participating to REPOSI approved the study. The attending physicians completed a standardized web-based case report form, including diagnosis at hospital admission, sociodemographic details and drug treatment at admission, during hospital stay and at discharge. In the second and third REPOSI runs, we decided to collect additional information and to do a short-term follow-up in order to improve the quality of data: main laboratory parameters, comorbidity according to the Cumulative Illness Rating Scale (CIRS) (Pamellee), basic activities of daily living, cognitive impairment, depression, and clinical events during hospital stay. Patients were then followed 3 months after discharge by means of a telephone interview in order to collect information on new diagnoses, hospital re-admissions, drug regimens, adverse events, and basic activities of daily living.

To establish the rate of potentially inappropriate XO inhibitor prescriptions, we considered all patients recruited in REPOSI, excluding only those who died in hospital or were transferred to another ward during hospitalization. To better evaluate the related risk of adverse clinical events, readmission and all-cause mortality, we considered only patients recruited in the second and third REPOSI run who had completed the 3-month follow-up. Of the 2318 in-patients discharged from hospital, follow-up data were not available for 585 (25\%), for various reasons: death before hospital discharge, transfer to another ward, refusal of the 3-month 
telephone interview, and discharge in critical conditions (life expectancy less than 3 month) or logistic reasons.

\section{Diseases and drug appropriateness}

Prescription of allopurinol or febuxostat was considered appropriate according to the presence of gout (including gout nephropathy), uric acid nephrolithiasis, and other manifestations like tophi (IDC-9 code 274). We also considered appropriate the prescription of allopurinol in patients with a diagnosis of chemotherapy-induced hyperuricemia (ICD-9 code 140-239 and diagnosis of hyperuricemia). The prescription of XO inhibitors was considered inappropriate in patients without the forementioned conditions. Dosage of XO inhibitors was not considered as criteria of drug appropriateness because this information was not collected. Diagnoses were derived by ICD-9 codes and personal interviews. In order to evaluate the predictors of inappropriate prescription of $\mathrm{XO}$ inhibitors, we compared the characteristics of patients considered inappropriately treated with those appropriately not treated (Fig. 1).

\section{Outcomes}

In order to evaluate the risk of adverse clinical events from discharge to the time of follow-up examination, re-admission, and all-cause mortality associated with inappropriate use of $\mathrm{XO}$ inhibitors, we considered eligible for this analysis only the patients with complete follow-up data. Information on readmission and survival of the patients was obtained after 3 months.

\section{Statistical analysis}

Analysis of variance was used to evaluate the relationship between potentially inappropriate use of XO inhibitors and incidence of adverse clinical events, re-admission, and mortality. Multivariate analyses were adjusted for age, sex, and possible confounders for CVD benefit of XO inhibitors. Analyses were done with JMP Pro 10 (SAS Institute Inc.).

\section{Results}

XO inhibitors were prescribed to 467 patients (11.6\%) among 4035 admitted to hospital and to 461 (13.2\%) among those discharged (3502). The mean number of drugs used and diagnoses were higher in patients given XO inhibitors than in those not treated (Table 1). Allopurinol was the most frequently prescribed XO inhibitor, only two patients at admission and two at discharge receiving febuxostat. At admission, only 39 patients $(8.4 \%$ of those receiving a XO inhibitor) and 43 at discharge $(9.3 \%)$ were appropriately treated;
428 patients (91.6\%) and $418(90.7 \%)$ were inappropriately treated at admission and discharge, respectively. Furthermore, 286 patients $(66.8 \%$ of patients inappropriately treated at admission) received a XO inhibitor inappropriately both at admission and discharge and only in 74 of them $(17.3 \%$ of patients inappropriately treated at admission) these drugs were appropriately withdrawn at the time of hospital discharge. In addition, among patients appropriately not treated at admission, the drug was inappropriately prescribed at discharge to 130 (31.1\% of patients inappropriately treated at discharge). The mean duration of XO inhibitor treatment was 3.3 years (interquartile range $0.9-8.3$ years, data available for 130 patients). Allopurinol was also inappropriately prescribed to five patients discharged in critical conditions.

Predictors of inappropriate prescription of $\mathrm{XO}$ inhibitors at discharge were evaluated by comparing the cohort of patients inappropriately treated $(n=418)$ with those appropriately not treated $(n=3,024)$. Among patients inappropriately treated, asymptomatic hyperuricemia, therapy with multiple drugs, chronic renal failure, diabetes, obesity, ischemic heart disease, heart failure, and cardiac arrhythmias were associated with greater prescription (Table 2). All these variables were independently associated with greater use of XO inhibitors in a multivariate model, adjusted for all the variables considered in the univariate model (data not shown).

The cohort of patients inappropriately treated and those appropriately not treated at discharge, which allowed to evaluate the association between XO inhibitors prescription and clinical outcomes at 3-month follow-up, included 2274 subjects and follow-up was available for 1697 (74.6\%). In them, prescription of XO inhibitors was associated with a higher risk of adverse clinical events, while no difference was found for the risk of re-hospitalization and all-cause mortality. Results were confirmed by multivariate analysis adjusted for age, sex, hyperuricemia, hypertension, diabetes, ischemic heart disease, heart failure, and cardiac arrhythmia (Table 3). Glomerular filtration rate (GFR) at discharge (estimated using the CKDEPI Creatinine Equation) was available for 1570 patients. Multivariate analysis adjusted for all previous covariates and GFR $<30 \mathrm{ml} / \mathrm{min} / 1.73 \mathrm{~m} \mathrm{[2]} \mathrm{found} \mathrm{similar} \mathrm{results} \mathrm{for} \mathrm{adverse}$ clinical events (odds ratio (OR) 1.94; $95 \%$ confidence interval (CI), 1.21-3.06; $p=0.0067$ ), re-admission (OR 0.67; $95 \% \mathrm{CI}$, $0.43-1.00 ; p=0.052$ ), and mortality (OR 1.20; $95 \%$ CI, 0.68 $2.02 ; p=0.52)$.

\section{Discussion}

This study provides a picture of elderly people with multimorbidity and polypharmacy currently treated in internal medical hospital wards with XO inhibitors, mainly allopurinol: approximately, $10-12 \%$, a prevalence similar to that observed in elderly patients living at home in New Zealand 


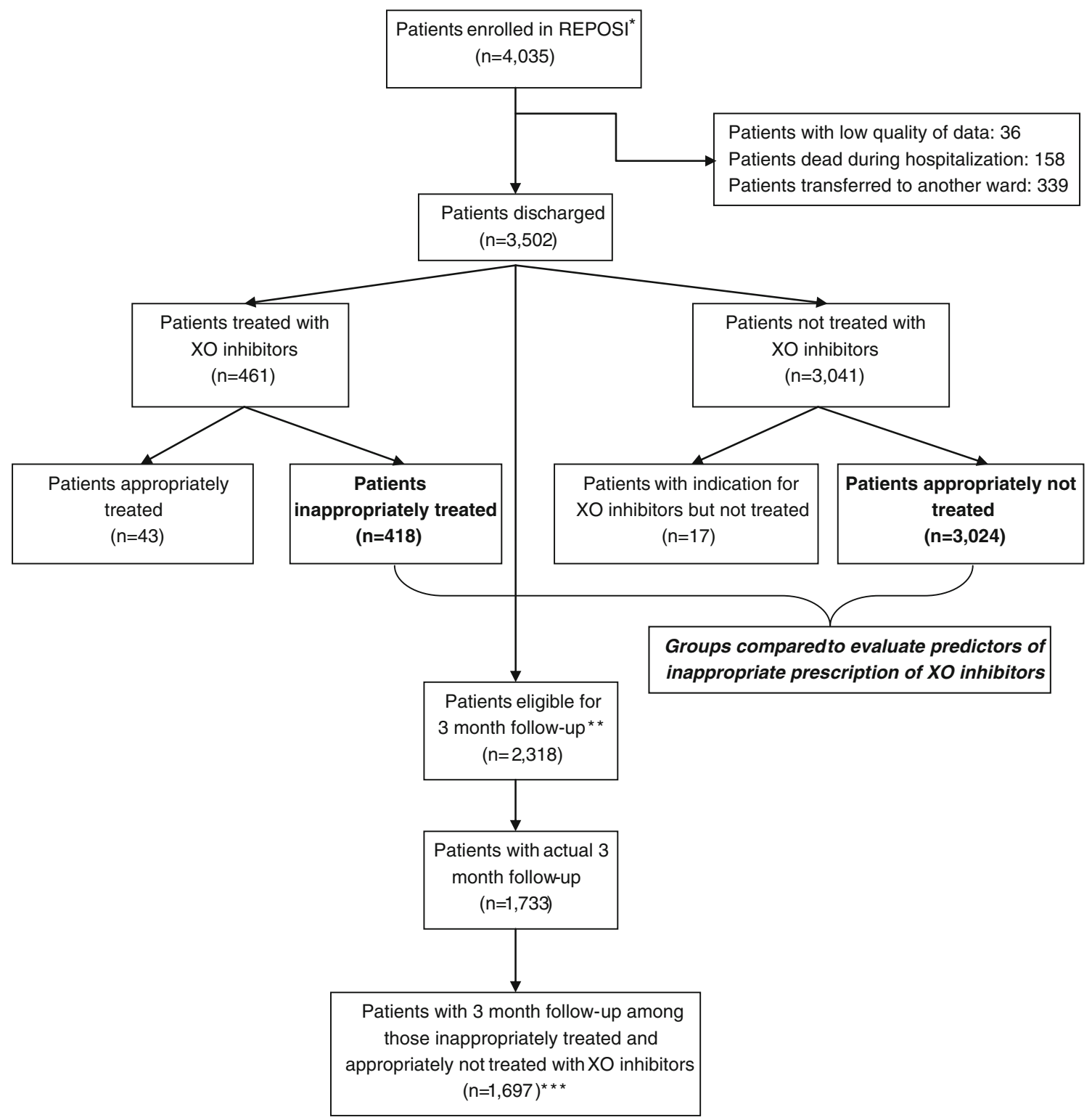

Fig. 1 Flow chart of the study

( $9 \%$ among patients between 65 and 74 years and $8 \%$ above 75 years) [10]. Hospital admission and discharge were both associated with a high prevalence of inappropriate prescription of allopurinol, mainly related to the management of hyperuricemia and various CVD. The observation that hospitalization was not associated with a reduction in the inappropriate use of allopurinol, particularly in hyperuricemic patients, is of special concern, because in this setting, confirmation of the inappropriate prescription did stem from expert hospital internists.
Table 1 Main characteristic of patients treated with xanthine oxidase (XO) inhibitors

\begin{tabular}{lllll}
\hline & At admission $(n=4,035)$ & \multicolumn{2}{l}{ At discharge $(n=3,502)$} \\
\hline & Treated & Not treated & Treated & Not treated \\
Total & 467 & 3,568 & 461 & 3,041 \\
Age, years (mean $\pm \mathrm{SD})$ & $79.5 \pm 7.0$ & $79.2 \pm 7.5$ & $79.0 \pm 7.1$ & $79.1 \pm 7.5$ \\
Women $(\%)$ & 39.5 & 53.4 & 41.4 & 54.0 \\
Drugs, no (mean $\pm \mathrm{SD})$ & $7.9 \pm 2.7$ & $4.9 \pm 2.7$ & $8.5 \pm 2.9$ & $5.9 \pm 2.8$ \\
Diagnoses, no $(\operatorname{mean} \pm \mathrm{SD})$ & $6.6 \pm 2.8$ & $5.1 \pm 2.7$ & $7.4 \pm 2.7$ & $6.1 \pm 2.7$ \\
\hline
\end{tabular}


Table 2 Predictors of inappropriate prescription of XO inhibitors at discharge

\begin{tabular}{|c|c|c|c|c|}
\hline & $\begin{array}{l}\text { Inappropriately } \\
\text { treated }\end{array}$ & $\begin{array}{l}\text { Appropriately not } \\
\text { treated }\end{array}$ & OR $(95 \% \mathrm{CI})$ & $p$ \\
\hline$n$ & 418 & 3,024 & & \\
\hline Drugs, no $(\text { mean } \pm \text { SD })^{\mathrm{a}}$ & $7.5 \pm 2.9$ & $5.9 \pm 2.8$ & & $<0.0001$ \\
\hline Age (years \pm SD) & $79.0 \pm 7.0$ & $79.1 \pm 7.5$ & & 0.50 \\
\hline Male $(\%)$ & 57.7 & 46.0 & $1.60(1.30-1.97)$ & $<0.0001$ \\
\hline Obesity (\%) & 6.0 & 3.2 & $1.94(1.23-3.05)$ & 0.007 \\
\hline Hypercholesterolemia (\%) & 8.9 & 11.3 & $0.76(0.53-1.09)$ & 0.12 \\
\hline Hypertension (\%) & 75.4 & 67.8 & $1.45(1.15-1.84)$ & 0.001 \\
\hline Diabetes $(\%)$ & 39.7 & 26.5 & $1.83(1.48-2.26)$ & $<0.0001$ \\
\hline Ischemic cardiomyopathy (\%) & 28.2 & 19.6 & $1.61(1.28-2.03)$ & $<0.0001$ \\
\hline Heart failure $(\%)$ & 34.0 & 16.3 & $2.63(2.11-3.30)$ & $<0.0001$ \\
\hline Cardiac dysrhythmias (\%) & 36.1 & 25.1 & $1.69(1.36-2.09)$ & $<0.0001$ \\
\hline Chronic renal failure (\%) & 43.8 & 13.3 & $5.06(4.07-6.31)$ & $<0.0001$ \\
\hline Asymptomatic hyperuricemia (\%) & 7.5 & 0.4 & $18.62(9.66-35.9)$ & $<0.0001$ \\
\hline Hypertension+ischemic cardiomyopathy+heart failure (\%) & 7.3 & 3.0 & $2.53(1.65-3.88)$ & $<0.0001$ \\
\hline Hypertension + diabetes + ischemic cardiomyopathy + heart failure $(\%)$ & 3.2 & 1.4 & $2.29(1.22-4.30)$ & 0.02 \\
\hline Polytherapy ${ }^{\mathrm{a}}(\%)$ & 87.1 & 66.9 & $3.33(2.48-4.47)$ & $<0.0001$ \\
\hline
\end{tabular}

${ }^{\text {a }}$ Prescription of $\geq 5$ drugs, excluding allopurinol or febuxostat

Why there is such a high prevalence of inappropriate prescription? Even though asymptomatic hyperuricemia affects $25-40 \%$ of patients with untreated hypertension, a recent Cochrane review suggests that there is insufficient evidence to recommend the use of allopurinol or other hypouricemic drugs as an initial or adjuvant treatment of hypertension [11]. In addition, results of two large prospective genetic cohort studies found no evidence for causal associations between uric acid levels and ischemic heart disease or high blood pressure [12]. Our findings of a high prescription rate contrast with the established knowledge that allopurinol has a potential for rare but life-threatening skin and systemic side effects. The risk of severe cutaneous adverse reactions is estimated to be 10 times higher in allopurinol users than in non-users: about $2 \%$ of

Table 3 Association between XO inhibitors and adverse clinical outcomes at 3 months follow-up

\begin{tabular}{lll}
\hline Univariate analysis & & \\
\hline & OR $(95 \%$ CI $)$ & $p$ value \\
Adverse clinical events & $1.68(1.10-2.58)$ & 0.02 \\
Re-admission & $0.84(0.57-1.23)$ & 0.36 \\
All-cause mortality & $1.30(0.80-2.12)$ & 0.30 \\
Multivariate analysis $^{\mathrm{a}}$ & & \\
Adverse clinical events & $1.74(1.09-2.70)$ & 0.02 \\
Re-admission & $0.73(0.48-1.08)$ & 0.12 \\
All-cause mortality & $1.22(0.71-2.03)$ & 0.46
\end{tabular}

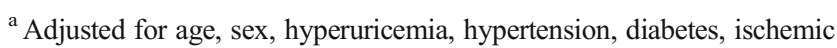
cardiomyopathy, heart failure, and cardiac dysrhythmias treated patients develop a skin rash. Furthermore, mortality from the severe hypersensitivity syndrome is not trivial, $0.2 / 1000$ patients dying every year following a severe adverse reaction to allopurinol $[13,14]$. The risk of fatal allopurinol hypersensitivity syndrome occurs more often after inappropriate treatment of asymptomatic hyperuricemia, especially in the first 6 months of treatment [7, 13]. A recent report from the center of pharmacovigilance of the Lombardy Region (Italy) showed that among 10 cases of adverse reactions to allopurinol reported over a period of 12 months, 7 had severe skin reactions, including the Stevens-Johnson syndrome and one patients died: in all cases allopurinol therapy was not consistent with the current guidelines for the treatment of asymptomatic hyperuricemia and gout [15]. Furthermore, a case of rhabdomyolysis following the administration of febuxostat for hyperuricemia has been reported in a patient with chronic kidney disease [16].

With this background, why allopurinol is so frequently prescribed? Assuming that prescription in asymptomatic hyperuricemic patients is meant to prevent an attack of gout, it must be considered that while the prevalence of hyperuricemia is about $5-8 \%$ in adult white males in the USA, only $5 \%$ of them will eventually develop gout [17]. It has been suggested that allopurinol could be of benefit for primary prevention in hypertensive and diabetic patients, especially those with left ventricular hypertrophy, and for secondary prevention in acute and chronic CVD. On the whole, available data indicate that the frequent choice to prescribe allopurinol to reduce various CVD in at risk populations is not supported by evidence, and must be balanced against rare but dangerous side effects. Indeed, no prospective randomized clinical trials 
have shown cardiovascular benefits on relevant clinical outcomes in the frame of primary or secondary prevention. This notwithstanding, we found in REPOSI a strict association between diagnosis of CVD and allopurinol prescription at hospital discharge, and although we cannot establish with certainty a causal relationship between a diagnosis of CVD and allopurinol prescription, our study suggests a high rate of inappropriate prescription of XO inhibitors for unlicensed indications. Unfortunately, at the 3-month follow-up, inappropriately treated patients had a higher risk for adverse events then those appropriately not treated and no benefits were found with respect to the risk of re-hospitalization and mortality. These results were consistent after adjusting for clinical conditions where a benefit for allopurinol has been suggested but not established (hyperuricemia, hypertension, diabetes, ischemic, heart failure, and cardiac dysrhythmias) and although patients receiving allopurinol were being on long-term treatment.

Our study has limitations: the REPOSI registry was not set up to specifically investigate the appropriate use of XO inhibitors but more generally to collect information on elderly hospital patients with multimorbidities receiving multiple drugs. Lack of data about uric acid levels made impossible to evaluate the use of allopurinol in those few patients with asymptomatic hyperuricemia who may have had a benefit, such as those with persistently high uric acid levels (above $13 \mathrm{mg} / \mathrm{dL}$ in men or $10 \mathrm{mg} / \mathrm{dL}$ in women) with the associated nephrotoxic effect [18]; or in the presence of urinary excretion of uric acid exceeding $1100 \mathrm{mg}$ daily, which has been associated with a $50 \%$ increase in the risk of developing uric acid stones $[19,20]$. Although this limitation tends to overestimate the rate of inappropriate prescription of $\mathrm{XO}$ inhibitors, it appears that for the majority of patients with asymptomatic hyperuricemia treatment with allopurinol is not justified by current evidences $[15,21]$. Study limitations also include the relatively smaller number of patients with 3-month follow-up data, not available for about $25 \%$ of those recruited. Moreover, no information was available on medication adherence, because we could not asses if a drug prescribed at hospital discharge was really taken. Lack of data on adverse clinical events and causes of death after discharge did limit the possibility of checking the association between XO inhibitors and adverse events, tending to underestimate the real risk.

\section{Conclusions}

The high prevalence of inappropriate prescription of $\mathrm{XO}$ inhibitors remained almost the same at admission and discharge. Inappropriate use of these drugs is principally related to treatment of asymptomatic hyperuricemia in patients with CVD, although no prospective randomized clinical trials have shown cardiovascular benefits. Stricter adherence to evidencebased guidelines is essential for a rational use of these drugs.
Acknowledgments REPOSI is a network of Italian internal medicine hospital wards which, voluntarily and without any financial support, agreed to participate in data collection during the four index weeks. Sponsor's role: none. We are grateful to J.D. Baggott for editorial assistance.

Conflict of interest The authors have no conflict of interest.

\section{Appendix}

Investigators and co-authors of the REPOSI (Registro Politerapie SIMI, Società Italiana di Medicina Interna) Study Group are as follows:

Steering Committee were as follows: Pier Mannuccio Mannucci (Chair, Fondazione IRCCS Cà Granda Ospedale Maggiore Policlinico, Milano), Alessandro Nobili (co-chair, IRCCS-Istituto di Ricerche Farmacologiche "Mario Negri", Milano), Mauro Tettamanti, Luca Pasina, Carlotta Franchi (IRCCSIstituto di Ricerche Farmacologiche "Mario Negri", Milano), Francesco Salerno (IRCCS Policlinico San Donato Milanese, Milano), Salvatore Corrao (ARNAS Civico, Di Cristina, Benfratelli, DiBiMIS, Università di Palermo, Palermo), Alessandra Marengoni (Spedali Civili di Brescia, Brescia), and Maura Marcucci (Dipartimento di Medicina Interna, Fondazione IRCCS Cà Granda Ospedale Maggiore Policlinico, Milano).

Clinical data monitoring and revision: Eleonora Sparacio, Stefania Alborghetti, Rosa Di Costanzo, Tarek Kamal Eldin (IRCCS-Istituto di Ricerche Farmacologiche "Mario Negri", Milano).

Database Management and Statistics: Mauro Tettamanti, Codjo Djignefa Djade (IRCCS-Istituto di Ricerche Farmacologiche "Mario Negri”, Milano) .

Investigators were as follows: Domenico Prisco, Elena Silvestri, Caterina Cenci, Tommaso Barnini (Azienda Ospedaliero Universitaria Careggi Firenze, SOD Patologia Medica); Giuseppe Delitala, Stefano Carta, Sebastiana Atzori (Azienda Mista Ospedaliera Universitaria, Sassari, Clinica Medica); Gianfranco Guarnieri, Michela Zanetti, Annalisa Spalluti (Azienda Ospedaliera Universitaria Ospedali Riuniti di Trieste, Trieste, Clinica Medica Generale e Terapia Medica); Maria Grazia Serra, Maria Antonietta Bleve (Azienda Ospedaliera "Cardinale Panico" di Tricase, Lecce, Unità Operativa Complessa Medicina); Massimo Vanoli, Giulia Grignani, Gianluca Casella (Azienda Ospedaliera della Provincia di Lecco, Ospedale di Merate, Lecco, Medicina Interna); Laura Gasbarrone (Azienda Ospedaliera Ospedale San Camillo Forlanini, Roma, Medicina Interna 1); Giorgio Maniscalco, Massimo Gunelli, Daniela Tirotta (Azienda Ospedaliera Ospedale San Salvatore, 
Pesaro, Soc Medicina Interna); Antonio Brucato, Silvia Ghidoni, Paola Di Corato (Azienda Ospedaliera Papa Giovanni XXIII, Bergamo, Medicina 1); Mauro Bernardi, Silvia Li Bassi, Luca Santi (Azienda Ospedaliera Policlinico Sant'Orsola-Malpighi, Bologna, Semeiotica Medica Bernardi); Giancarlo Agnelli, Alfonso Iorio, Maura Marcucci, Emanuela Marchesini (Azienda Ospedaliera Santa Maria della Misericordia, Perugia, Medicina Interna e Cardiovascolare); Elmo Mannarino, Graziana Lupattelli, Pamela Rondelli, Francesco Paciullo (Azienda Ospedaliera Santa Maria della Misericordia, Perugia, Medicina Interna, Angiologia, Malattie da Arteriosclerosi); Fabrizio Fabris, Michela Carlon, Francesca Turatto (Azienda Ospedaliera Università di Padova, Padova, Clinica Medica I); Maria Cristina Baroni, Marianna Zardo (Azienda Ospedaliera Università di Parma, Parma, Clinica e Terapia Medica); Roberto Manfredini, Christian Molino, Marco Pala, Fabio Fabbian (Azienda Ospedaliera-Universitaria Sant'Anna, Ferrara, Unità Operativa Clinica Medica); Ranuccio Nuti, Roberto Valenti, Martina Ruvio, Silvia Cappelli (Azienda Ospedaliera Università Senese, Siena, Medicina Interna I); Giuseppe Paolisso, Maria Rosaria Rizzo, Maria Teresa Laieta (Azienda Ospedaliera Universitaria della Seconda Università degli Studi di Napoli, Napoli, VI Divisione di Medicina Interna e Malattie Nutrizionali dell'Invecchiamento); Teresa Salvatore, Ferdinando Carlo Sasso (Azienda Ospedaliera Universitaria della Seconda Università degli Studi di Napoli, Napoli, Medicina Interna e Malattie Epato-Bilio Metaboliche Avanzate); Riccardo Utili, Emanuele Durante Mangoni, Daniela Pinto (Azienda Ospedaliera Universitaria della Seconda Università degli Studi di Napoli, Napoli, Medicina Infettivologica e dei trapianti); Oliviero Olivieri, Anna Maria Stanzial (Azienda Ospedaliera Universitaria Integrata di Verona, Verona, Unità Operativa di Medicina Interna B); Renato Fellin, Stefano Volpato, Sioulis Fotini (Azienda Ospedaliera Universitaria Ospedale Sant'Anna, Ferrara, Unità Operativa di Medicina Interna Gerontologia e Geriatria); Mario Barbagallo, Ligia Dominguez, Lidia Plances, Daniela D'Angelo (Azienda Ospedaliera Universitaria Policlinico Giaccone Policlinico di Palermo, Palermo, Unità Operativa di Geriatria e Lungodegenza); Giovanbattista Rini, Pasquale Mansueto, Ilenia Pepe (Azienda Ospedaliera Universitaria Policlinico P. Giaccone di Palermo, Palermo, Medicina Interna e Malattie Metaboliche); Giuseppe Licata, Luigi Calvo, Maria Valenti (Azienda Ospedaliera Universitaria Policlinico P. Giaccone di Palermo, Palermo, Medicina Interna e Cardioangiologia); Claudio Borghi, Enrico Strocchi, Elisa Rebecca Rinaldi (Azienda Ospedaliera Universitaria Policlinico S. Orsola-Malpighi, Bologna,
Unità Operativa di Medicina Interna Borghi); Marco Zoli, Elisa Fabbri, Donatella Magalotti (Azienda Ospedaliera Universitaria Policlinico S. Orsola-Malpighi, Bologna, Unità Operativa di Medicina Interna Zoli); Alberto Auteri, Anna Laura Pasqui, Luca Puccetti (Azienda Ospedaliera Universitaria Senese, Siena, Medicina 3); Franco Laghi Pasini, Pier Leopoldo Capecchi, Maurizio Bicchi (Azienda Ospedaliera Universitaria Senese, Siena, Unità Operativa Complessa Medicina 2); Carlo Sabbà, Francesco Saverio Vella, Alessandro Marseglia, Chiara Valentina Luglio (Azienda Ospedaliero-Universitaria Consorziale Policlinico di Bari, Bari, Medicina Interna Universitaria C. Frugoni); Giuseppe Palasciano, Maria Ester Modeo, Annamaria Aquilino, Pallante Raffaele (Azienda OspedalieroUniversitaria Consorziale Policlinico di Bari, Bari, Medicina Interna Ospedale "Pende-Ferrannini"); Stefania Pugliese, Caterina Capobianco (Azienda OspedalieroUniversitaria Consorziale Policlinico di Bari, Bari, Clinica Medica I Augusto Murri); Alfredo Postiglione, Maria Rosaria Barbella, Francesco De Stefano (Azienda Ospedaliera Universitaria Policlinico Federico II di Napoli, Medicina Geriatrica Dipartimento di Clinica Medica); Luigi Fenoglio, Chiara Brignone, Christian Bracco, Alessia Giraudo (Azienda Sanitaria Ospedaliera Santa Croce e Carle di Cuneo, Cuneo, S. C. Medicina Interna); Giuseppe Musca, Olga Cuccurullo (Azienda Sanitaria Provinciale di Cosenza Presidio Ospedaliero di Cetraro, Cosenza, Unità Operativa Complessa Medicina Interna); Luigi Cricco, Alessandra Fiorentini (COB Stabilimento Montefiascone, Viterbo, Unità Operativa Complessa di Geriatria e Medicina); Maria Domenica Cappellini, Giovanna Fabio, Sonia Seghezzi, Margherita Migone De Amicis (Fondazione IRCCS Cà Granda Ospedale Maggiore Policlinico, Milano, Unità Operativa Medicina Interna IA); Silvia Fargion, Paola Bonara, Mara Bulgheroni, Rosa Lombardi (Fondazione IRCCS Cà Granda Ospedale Maggiore Policlinico, Milano, Medicina Interna 1B); Fabio Magrini, Ferdinando Massari, Tatiana Tonella (Fondazione IRCCS Cà Granda Ospedale Maggiore Policlinico, Milano, Unità Operativa Medicina Cardiovascolare); Flora Peyvandi, Alberto Tedeschi, Raffaella Rossio (Fondazione IRCCS Cà Granda Ospedale Maggiore Policlinico, Milano, Medicina Interna 2); Guido Moreo, Barbara Ferrari, Luisa Roncari (Fondazione IRCCS Cà Granda Ospedale Maggiore Policlinico, Milano, Medicina Interna 3); Valter Monzani, Valeria Savojardo, Christian Folli, Maria Magnini (Fondazione IRCCS Cà Granda Ospedale Maggiore Policlinico, Milano, Medicina d'Urgenza); Daniela Mari, Paolo Dionigi Rossi, Sarah Damanti, Silvia Prolo (Fondazione IRCCS Cà Granda Ospedale Maggiore Policlinico, Milano, Geriatria); Maria Sole 
Lilleri (Fondazione IRCCS Cà Granda Ospedale Maggiore Policlinico, Milano, Medicina Generale ad Indirizzo Geriatrico); Luigi Cricco, Alessandra Fiorentini (COB Viterbo, Stabilimento Montefiascone, Viterbo, UOC Geriatria e Medicina); Giuliana Micale (IRCCS Istituto Auxologico Italiano, Milano, Medicina Generale ad indirizzo Geriatrico); Mauro Podda, Carlo Selmi, Francesca Meda (IRCCS Istituto Clinico Humanitas, Milano, Clinica Medica); Francesco Salerno, Silvia Accordino, Alessio Conca, Valentina Monti (IRCCS Policlinico San Donato e Università di Milano, San Donato Milanese, Medicina Interna); Gino Roberto Corazza, Emanuela Miceli, Marco Vincenzo Lenti, Donatella Padula (IRCCS Policlinico San Matteo di Pavia, Pavia, Clinica Medica I, Reparto 11); Carlo L. Balduini, Giampiera Bertolino, Stella Provini, Federica Quaglia (IRCCS Policlinico San Matteo di Pavia, Pavia, Clinica Medica III); Giovanni Murialdo, Marta Bovio (IRCS Azienda Ospedaliera Universitaria San MartinoIST di Genova, Genova, Clinica di Medicina Interna 2); Franco Dallegri, Luciano Ottonello, Alessandra Quercioli, Alessandra Barreca (Università di Genova, Genova, Medicina Interna 1); Maria Beatrice Secchi, Davide Ghelfi (Ospedale Bassini di Cinisello Balsamo, Milano, Divisione Medicina); Wu Sheng Chin, Laura Carassale, Silvia Caporotundo (Ospedale Bassini, Cinisello Balsamo, Milano, Unità Operativa di Geriatria); Luigi Anastasio, Lucia Sofia, Maria Carbone (Ospedale Civile Jazzolino di Vibo Valentia, Vibo Valentia, Medicina interna); Giancarlo Traisci, Lucrezia De Feudis, Silvia Di Carlo (Ospedale Civile Santo Spirito di Pescara, Pescara, Medicina Interna 2); Giovanni Davì, Maria Teresa Guagnano, Simona Sestili (Ospedale Clinicizzato SS. Annunziata, Chieti, Clinica Medica); Elisabetta Bergami, Emanuela Rizzioli (Ospedale del Delta, Lagosanto, Ferrara, Medicina Interna); Carlo Cagnoni, Luca Bertone, Antonio Manucra (Ospedale di Bobbio, Piacenza, Unità Operativa Medicina e Primo Soccorso); Alberto Buratti, Tiziana Tognin, Nicola Lucio Liberato (Azienda Ospedaliera della Provincia di Pavia, Ospedale di Casorate Primo, Pavia, Medicina Interna); Giordano Bernasconi, Barbara Nardo (Ospedale di Circolo di Busto Arsizio, Varese, Medicina I); Giovanni Battista Bianchi, Sabrina Giaquinto Ospedale "SS Gerosa e Capitanio" di Lovere, Bergamo, Unità Operativa Complessa di Medicina Generale, Azienda Ospedaliera "Bolognini" di Seriate, Bergamo; Giampiero Benetti, Michela Quagliolo, Giuseppe Riccardo Centenaro (Ospedale di Melegnano, Vizzolo Predabissi, Melegnano, Medicina 1); Francesco Purrello, Antonino Di Pino, Salvatore Piro (Ospedale Garibaldi Nesima, Catania, Unità Operativa Complessa di Medicina Interna); Gerardo Mancuso, Daniela Calipari, Mosè Bartone, Francesco Gullo
(Ospedale Giovanni Paolo II Lamezia Terme, Catanzaro, Unità Operativa Complessa Medicina Interna); Michele Cortellaro, Marina Magenta, Francesca Perego; Maria Rachele Meroni (Ospedale Luigi Sacco, Milano, Medicina $3^{\circ}$ ); Marco Cicardi, Antonio Gidaro Marina Magenta (Ospedale Luigi Sacco, Milano, Medicina II); Andrea Sacco, Antonio Bonelli, Gaetano Dentamaro (Ospedale Madonna delle Grazie, Matera, Medicina); Renzo Rozzini, Lina Falanga, Alessandro Giordano (Ospedale Poliambulanza, Brescia, Medicina Interna e Geriatria); Paolo Cavallo Perin, Bartolomeo Lorenzati, Gabriella Gruden, Graziella Bruno (Dipartimento di Scienze Mediche, Università di Torino, Città della Scienza e della Salute, Torino, Medicina 3); Giuseppe Montrucchio, Elisabetta Greco, Pietro Tizzani (Dipartimento di Scienze Mediche, Università di Torino, Città della Scienza e della Salute, Torino, Medicina Interna 5); Giacomo Fera, Maria Loreta Di Luca, Donatella Renna (Ospedale San Giacomo di Monopoli, Bari, Unità Operativa Medicina Interna); Antonio Perciccante, Alessia Coralli (Ospedale San GiovanniDecollato-Andisilla, Civita Castellana Medicina); Rodolfo Tassara, Deborah Melis, Lara Rebella (Ospedale San Paolo, Savona, Medicina I); Giorgio Menardo, Stefania Bottone, Elsa Sferrazzo (Ospedale San Paolo, Savona, Medicina Interna e Gastroenterologia); Claudio Ferri, Rinaldo Striuli, Rosa Scipioni (Ospedale San Salvatore, L'Aquila, Medicina Interna Universitaria); Raffaella Salmi, Piergiorgio Gaudenzi, Susanna Gamberini, Franco Ricci (Azienda OspedalieraUniversitaria S. Anna, Ferrara, Unità Operativa di Medicina Ospedaliera II); Cosimo Morabito, Roberto Fava (Ospedale Scillesi d'America, Scilla Medicina); Andrea Semplicini, Lucia Gottardo (Ospedale SS. Giovanni e Paolo, Venezia, Medicina Interna 1); Giuseppe Delitala, Stefano Carta, Sebastiana Atzori (Ospedale Universitario Policlinico di Sassari, Sassari, Clinica Medica); Gianluigi Vendemiale, Gaetano Serviddio, Roberta Forlano (Ospedali Riuniti di Foggia, Foggia, Medicina Interna Universitaria); Luigi Bolondi, Leonardo Rasciti, Ilaria Serio (Policlinico Sant'OrsolaMalpighi, Bologna, Unità Operativa Complessa Medicina Interna); Cesare Masala, Antonio Mammarella, Valeria Raparelli (Policlinico Umberto I, Roma, Medicina Interna D); Filippo Rossi Fanelli, Massimo Delfino, Antonio Amoroso (Policlinico Umberto I, Roma, Medicina Interna H); Francesco Violi, Stefania Basili, Ludovica Perri (Policlinico Umberto I, Roma, Prima Clinica Medica); Pietro Serra, Vincenzo Fontana, Marco Falcone (Policlinico Umberto I, Roma, Terza Clinica Medica); Raffaele Landolfi, Antonio Grieco, Antonella Gallo (Policlinico Universitario A. Gemelli, Roma, Clinica Medica); Giuseppe Zuccalà, Francesco 
Franceschi, Guido De Marco, Cordischi Chiara, Sabbatini Marta (Policlinico Universitario A. Gemelli, Roma, Roma, Unità Operativa Complessa Medicina d'Urgenza e Pronto Soccorso); Martino Bellusci, Donatella Setti, Filippo Pedrazzoli (Presidio Ospedaliero Alto Garda e Ledro, Ospedale di Arco, Trento, Unità Operativa di Medicina Interna Urgenza/Emergenza); Giuseppe Romanelli, Caterina Pirali, Claudia Amolini (Spedali Civili di Brescia, Brescia, Geriatria); Enrico Agabiti Rosei, Damiano Rizzoni, Luana Castoldi (Spedali Civili di Brescia, Brescia, Seconda Medicina); Antonio Picardi, Umberto Vespasiani Gentilucci, Chiara Mazzarelli, Paolo Gallo (Università Campus Bio-Medico, Roma, Medicina Clinica-Epatologia); Luigina Guasti, Luana Castiglioni, Andrea Maresca, Alessandro Squizzato, Sara Contini, Marta Molaro (Università degli Studi dell'Insubria, Ospedale di Circolo e Fondazione Macchi, Varese, Medicina Interna I); Giorgio Annoni, Maurizio Corsi, Sara Zazzetta (Università degli studi di Milano-Bicocca Ospedale S. Gerardo, Monza, Unità Operativa di Geriatria); Marco Bertolotti, Chiara Mussi, Roberto Scotto, Maria Alice Ferri, Francesca Veltri (Università di Modena e Reggio Emilia, AUSL di Modena, Modena, Nuovo Ospedale Civile, Unità Operativa di Geriatria); Franco Arturi, Elena Succurro, Giorgio Sesti, Umberto Gualtieri (Università degli Studi Magna Grecia, Policlinico Mater Domini, Catanzaro, Unità Operativa Complessa di Medicina Interna); Francesco Perticone, Angela Sciacqua, Michele Quero, Chiara Bagnato (Università Magna Grecia Policlinico Mater Domini, Catanzaro, Unità Operativa Malattie Cardiovascolari Geriatriche); Paola Loria, Maria Angela Becchi, Gianfranco Martucci, Alessandra Fantuzzi, Mauro Maurantonio (Università di Modena e Reggio Emilia, Medicina Metabolica-NOCSAE, Baggiovara, Modena); Roberto Corinaldesi, Roberto De Giorgio, Mauro Serra, Valentina Grasso, Eugenio Ruggeri, Lorenzo Mauro Carozza, Fabio Pignatti (Dipartimento di Scienze Mediche e Chirurgiche, Unità Operativa di Medicina Interna, Università degli Studi di Bologna/Azienda OspedalieroUniversitaria S. Orsola-Malpighi, Bologna).

\section{References}

1. Struthers A, Shearer F (2012) Allopurinol: novel indications in cardiovascular disease. Heart 98(21):1543-1545

2. Higgins P, Dawson J, Lees KR et al (2012) Xanthine oxidase inhibition for the treatment of cardiovascular disease: a systematic review and meta-analysis. Cardiovasc Ther 30(4):217-226
3. Luk AJ, Levin GP, Moore EE et al (2009) Allopurinol and mortality in hyperuricaemic patients. Rheumatology 48(7):804-806

4. Thanassoulis G, Brophy JM, Richard H et al (2010) Gout, allopurinol use, and heart failure outcomes. Arch Intern Med 9;170(15):13581364

5. Rentoukas E, Tsarouhas K, Tsitsimpikou C et al (2010) The prognostic impact of allopurinol in patients with acute myocardial infarction undergoing primary percutaneous coronary intervention. Int $\mathrm{J}$ Cardiol 145:257e8

6. Goicoechea M, de Vinuesa SG, Verdalles U et al (2010) Effect of allopurinol in chronic kidney disease progression and cardiovascular risk. Clin J Am Soc Nephrol 5:1388e93

7. Gutiérrez-Macías A, Lizarralde-Palacios E, Martínez-Odriozola $P$ et al (2005) Fatal allopurinol hypersensitivity syndrome after treatment of asymptomatic hyperuricaemia. BMJ 17;331(7517): 623-624

8. Khanna D, Fitzgerald JD, Khanna PP et al (2012) American College of Rheumatology. American College of Rheumatology Guidelines for Management of Gout: part 1, systematic nonpharmacologic and pharmacologic therapeutic approaches to hyperuricemia. Arthritis Care Res 64(10):1431-1446

9. White WB, Chohan S, Dabholkar A et al (2012) Cardiovascular safety of febuxostat and allopurinol in patients with gout and cardiovascular comorbidities. Am Heart J 164(1):14-20

10. Horsburgh S, Norris P, Becket G et al (2014) Allopurinol use in a New Zealand population: prevalence and adherence. Rheumatol Int 34(7):963-970

11. Gois PH, Souza ER (2013) Pharmacotherapy for hyperuricemia in hypertensive patients. Cochrane Database Syst Rev 1:CD008652

12. Palmer TM, Nordestgaard BG, Benn M et al (2013) Association of plasma uric acid with ischaemic heart disease and blood pressure: mendelian randomisation analysis of two large cohorts. BMJ 18;347: f4262

13. Kim SC, Newcomb C, Margolis D et al (2013) Severe cutaneous reactions requiring hospitalization in allopurinol initiators: a population-based cohort study. Arthritis Care Res 65(4):578584

14. Singer JZ, Wallace SL (1986) The allopurinol hypersensitivity syndrome. Unnecessary morbidity and mortality. Arthritis Rheum 29(1): $82-87$

15. Carnovale, C, Venegoni M, Clementi E. Allopurinol Overuse in Asymptomatic Hyperuricemia A Teachable Moment. JAMA Intern Med 2014. doi:10.1001/jamainternmed.2014.1427

16. Kang Y, Kim MJ, Jang HN et al (2014) Rhabdomyolysis associated with initiation of febuxostat therapy for hyperuricaemia in a patient with chronic kidney disease. J Clin Pharm Ther 39(3): $328-330$

17. Becker MA (1988) Clinical aspects of monosodium urate monohydrate crystal deposition disease (gout). Rheum Dis Clin N Am 14:377

18. Dincer HE, Dincer AP, Levinson DJ (2002) Asymptomatic hyperuricemia: to treat or not to treat. Cleve Clin J Med 69(8):594-608, 597, $600-602$

19. Kjellstrand CM, Cambell DC II, von Hartitzsch B et al (1974) Hyperuricemic acute renal failure. Arch Intern Med 133(3):349-359

20. Ettinger B, Tang A, Citron JT et al (1986) Randomized trial of allopurinol in the prevention of calcium oxalate calculi. N Engl J Med 315(22):1386-1389

21. Mikuls TR, Farrar JT, Bilker WB et al (2005) Suboptimal physician adherence to quality indicators for the management of gout and asymptomatic hyperuricaemia: results from the UK General Practice Research Database (GPRD). Rheumatology 44(8):10381042 